\title{
Exploring the Valence Electron Distribution in High Temperature Superconductors with a Focused Electron Probe
}

\author{
J. Tafto ${ }^{* * *}$, Lijun $\mathrm{Wu}^{*}$ and Yimei Zhu* \\ * Materials Science Department, Brookhaven National Laboratory, Upton, NY11973, USA \\ *** Department of Physics, University of Oslo, P.O. Box 1048 Blindern, 0316 Oslo, Norway
}

Since the discovery of the high temperature superconductors there has been a great deal of experimental and theoretical effort to relate their superconducting properties and electronic structure. We have used a focused $300 \mathrm{keV}$ electron probe to study important features associated with the electronic structure of these oxides, in particular electron transfer away from the $\mathrm{CuO}_{2}$ planes that creates the electron holes that are believed to be responsible for the superconductivity of these oxides. Our experimental procedure differs from conventional convergent electron diffraction, $\mathrm{CBED}$, in that we focus the electron probe some 100 micrometers above the specimen, rather than at the specimen level. Thus, when the beam divergence is small enough to avoid overlap between the diffraction disks, each diffraction disk becomes a dark-field shadow image of the specimen. We use wedge-shaped thin specimens, enabling us to record the intensity variation with thickness for many reflections at the same time. We refer to this method as PArallel Recording Of Dark-field Images, PARODI [1,2].

Before starting with the cuprates, we first take a look at the recently discovered superconductor $\mathrm{MgB}_{2}$. Fig. 1 shows conventional CBED patterns and fig. 2 shows PARODI patterns of the $00 \mathrm{l}$ row where the planar spacing is $0.352 \mathrm{~nm}$. For this material conventional CBED and PARODI give virtually the same value and accuracy for the structure factor of the 001 reflection. Given the small unit cell and the low $\mathrm{Z}$ elements of $\mathrm{MgB}_{2}$, this material is also well suited for $\mathrm{x}$-ray diffraction using a synchrotron beam-line [3]. From powder x-ray diffraction experiments, many structure factors, and thus sufficient data for a electron density map is available from a single experiment. Nonetheless quantitative electron diffraction is an important supplement in that, for selected structure factors, very high accuracy can be achieved using CBED and PARODI. Measuring just a few of these provides a useful test of electronic structure calculations.

Electron diffraction is particular powerful for reflections with short g-vectors that occur for crystals with large unit cells. The reason is that the electrons interact with the electrostatic potential, and it follows from the Mott formula that for short g-vectors the Fourier components of the electrostatic potential are extremely sensitive to the valence electron distribution. This sensitivity is clearly illustrated by considering $\mathrm{Bi}_{2} \mathrm{Sr}_{2} \mathrm{CaCu}_{2} \mathrm{O}_{8+\delta}$. The innermost, i.e. 002, reflection in this material corresponds to a planar spacing of $1.54 \mathrm{~nm}$. Calculated values of the x-ray structure factor with the origin chosen at the $\mathrm{Ca}$ atom is $-150.7 \mathrm{e}$ based on the ionic model and $-157.9 \mathrm{e}$ from electronic structure calculations [4]. Converting these structure factors to electron structure factors gives $147.9 \AA$ and $15.8 \AA$, respectively i.e. they differ by a factor 10 in absolute values for the two models, and their signs are opposite. Fortunately dynamical electron diffraction patterns also provide information about the phase of the structure factors.

Conventional CBED is not easily applied to dense reciprocal lattice rows because the angle spanned by the convergent beam disks has to be very small to avoid overlap between them. On the other hand PARODI works well because we then study the intensity variation with thickness, and the thickness range in the shadow images varies with the distance from the focused probe to the specimen. Fig 3 
shows a PARODI pattern for the (001) row of $\mathrm{Bi}_{2} \mathrm{Sr}_{2} \mathrm{CaCu}_{2} \mathrm{O}_{8+\delta}$. Based on such patterns we arrived, for the 002 electron structure factor, at the value $15 \pm 4 \AA$ [2]. After conversion to x-ray structure factor this becomes $-157.9 \pm 0.2 \mathrm{e}$ which is the same as from electronic structure calculations.

\section{References}

[1] J. Tafto, Y. Zhu and L. Wu, Acta Cryst. A 54, 532 (1998)

[2] L. Wu, Y. Zhu and J. Tafto, Phys. Rev. B 59, 6035 (1999)

[3] E. Nishibori et. al. J. Phys. Soc. Japan. 70, 2252 (2001)

[4] R. P. Gupta and M. Gupta, Phys. Rev. B 49, 13154 (1994)

[5] Work supported by US DOE under contract No DE-AC02-98CH10886 and the Norwegian Research Council under the FIN project.

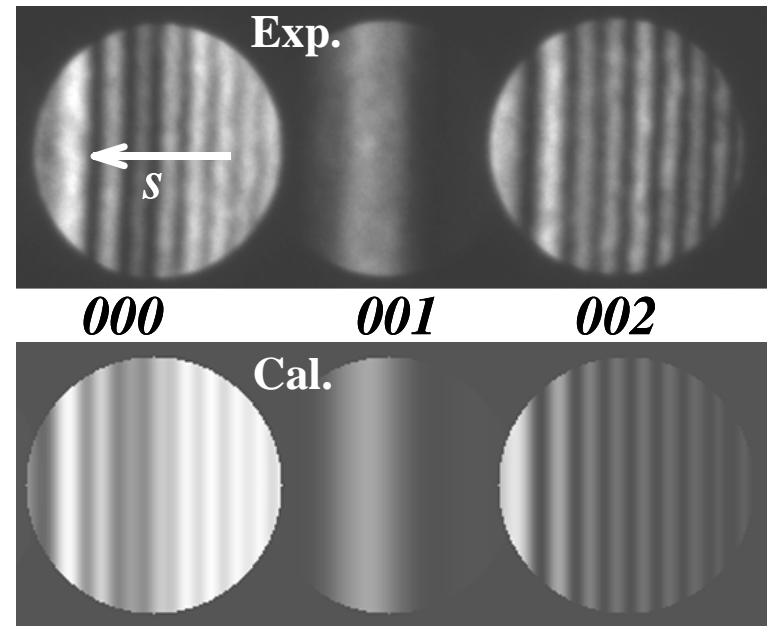

FIG.1. Experimental and calculated CBED pattern of $\mathrm{MgB}_{2}$
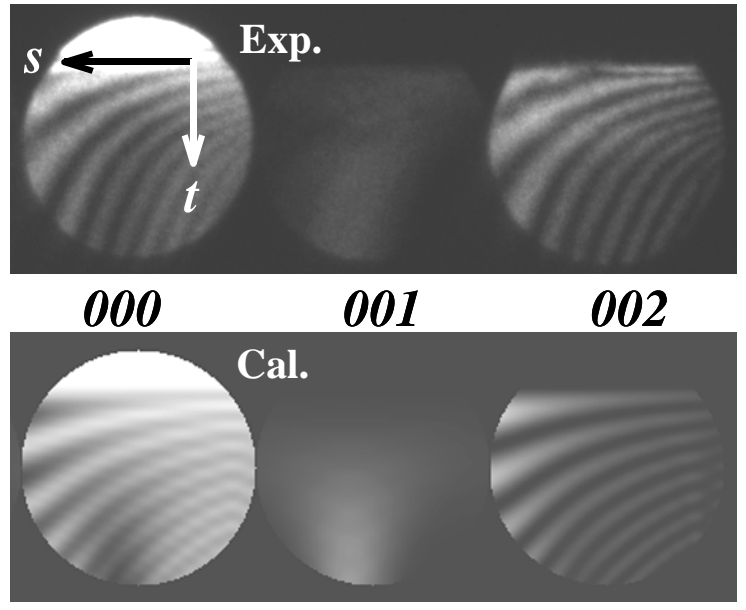

FIG.2. Experimental and calculated PARODI pattern of $\mathrm{MgB}_{2}$.

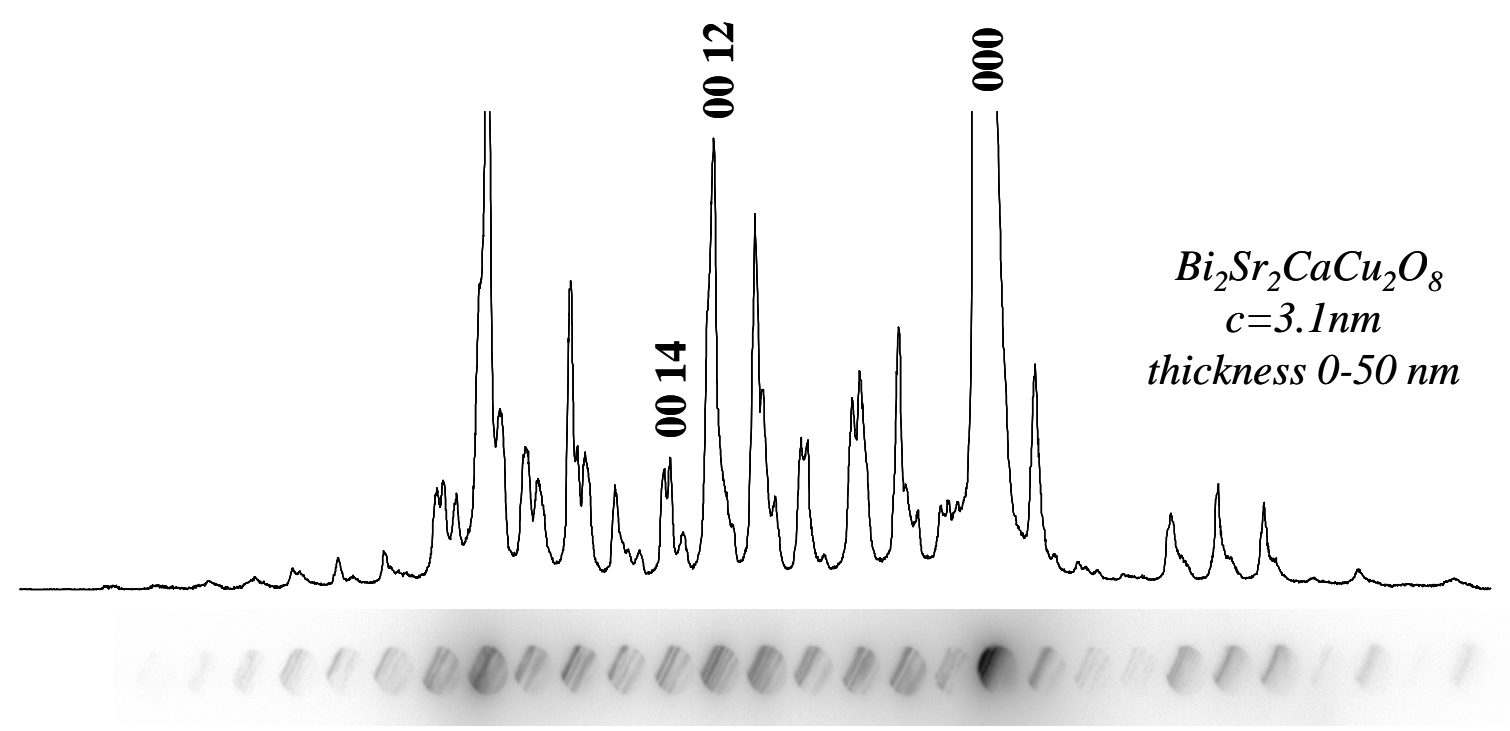

FIG.3. Experimental PARODI pattern of the (001) reciprocal row of $\mathrm{Bi}_{2} \mathrm{Sr}_{2} \mathrm{CaCu}_{2} \mathrm{O}_{8+\delta}$. 